\title{
Management of the multiple symptoms of irritable bowel syndrome
}

\author{
Magnus Simrén, Hans Törnblom, Olafur S Palsson, William E Whitehead
}

Lancet Gastroenterol Hepatol 2017; 2: 112-22

Department of Internal Medicine and Clinical Nutrition, Institute of Medicine, Sahlgrenska Academy, University of Gothenburg, Gothenburg, Sweden (Prof M Simrén MD, HTörnblom MD); and Center for Functional Gastrointestinal and Motility Disorders, University of North Carolina at Chapel Hill, Chapel Hill, NC, USA (Prof O S Palsson PsyD, ProfW EWhitehead PhD, Prof M Simrén)

Correspondence to: Prof Magnus Simrén, Department of Internal Medicine and Clinical Nutrition, Institute of Medicine, Sahlgrenska Academy, University of Gothenburg,

41345 Gothenburg, Sweden magnus.simren@medicine.gu.

Irritable bowel syndrome (IBS) is one of the most common functional gastrointestinal disorders. A stepwise management approach is advocated for patients with IBS. For a substantial proportion of patients with mild symptoms, general management principles, including making a confident diagnosis and offering explanation, reassurance, and dietary and lifestyle advice, are sufficient. However, many patients continue to have moderate-to-severe symptoms and are not satisfied solely with this approach. In these patients, use of pharmacotherapy on the basis of the predominant symptom (constipation, diarrhoea, pain, or bloating) or combination of symptoms is the next step. For patients with symptoms that are refractory to these initial treatment options and those who have comorbid conditions or psychological symptoms, a combination of therapies should be used, and the use of psychotropic drugs and psychological treatment alternatives is often effective. Finally, the key to successful treatment of patients with IBS is a good physician-patient relationship and use of person-centred care principles.

\section{Introduction}

Irritable bowel syndrome (IBS) is one of the most common functional gastrointestinal disorders, characterised by abdominal pain associated with abnormal bowel habits. There is no valid biomarker for IBS; diagnosis relies on the presence of characteristic symptoms defined by symptom-based diagnostic criteria, the most recent being the Rome IV criteria, and the absence of objective findings from a limited number of standard diagnostic tests and investigations. ${ }^{1}$ Prevalence varies substantially depending on the investigations and criteria used to define IBS, but a 2012 meta-analysis ${ }^{2}$ showed a pooled worldwide prevalence of $11 \cdot 2 \%$. IBS is more common in women than in men, and occurs in children as well as adults of all ages. Prevalence tends to decline with increasing age. ${ }^{2}$ Health-care use is high for people with IBS, and their work productivity is reduced, which leads to high costs for society. ${ }^{3}$ Most patients with IBS are managed in primary care, but a substantial proportion also seek health care with gastroenterologists. The decision to seek health care is associated with the severity of both gastrointestinal and extra-intestinal symptoms, and psychological factors also seem to be an important determinant., ${ }^{4,5}$

A major challenge in the management of IBS is the fact that the predominant symptoms vary substantially from person to person. IBS is characterised by a combination of abdominal pain and abnormal bowel habits: some patients have predominantly loose and frequent stools (IBS with diarrhoea, IBS-D); others, infrequent and hard stools (IBS with constipation, IBS-C); and a third group alternates between diarrhoea and constipation (IBS mixed, IBS-M). ${ }^{1}$ Other prevalent gastrointestinal symptoms in patients with IBS are bloating and abdominal distension, and symptoms thought to emanate from parts of the gastrointestinal tract other than the bowel. A substantial proportion of patients with IBS have overlapping upper and lower gastrointestinal symptoms. ${ }^{6}$ Moreover, extra-intestinal symptoms (ie, back pain; headaches; urogenital symptoms; sleep disturbances; functional syndromes such as fibromyalgia, temporomandibular joint syndrome, and chronic fatigue syndrome; and psychological comorbidity) are common in patients with IBS. In fact, the presence of these extra-intestinal symptoms seems to be as important as the presence of gastrointestinal symptoms in predicting burden of illness, health outcome, and quality of life in patients with IBS, and therefore it is important to identify and manage these symptoms effectively. ${ }^{8}$ Despite IBS not being life-threatening or associated with severe medical complications, patients with this disorder often report profound reductions in quality of life, similar to many medically more severe diseases. ${ }^{9}$

Current treatment options for IBS are limited; recommendations about management strategies include general measures directed to all patients, and more targeted actions based on the symptom profile of the patient. ${ }^{10}$ In this Review, we discuss general treatment and management principles for patients with IBS, but the main focus will be on how to address the specific symptoms that need more targeted treatment approaches in patients who are not responding sufficiently to conservative, general treatment.

\section{General treatment and management principles}

A key factor for successful management of patients with IBS is to establish a good physician-patient relationship. This includes adequately addressing patient concerns, involving the patient in the approach to diagnosis and management, addressing the factors that precipitated health-care seeking, offering continuity, setting realistic goals, and performing a cost-effective evaluation. Optimising interactions between the health-care provider and the patient and using a person-centred approach are likely to enhance the outcome for the patient. ${ }^{11}$

In general, a stepwise management approach is advocated, since some patients need only explanation and reassurance, whereas other need more extensive treatment options (figure 1). When deciding which treatment options to choose, several factors should be taken into account to create a multidimensional clinical 
profi le of the patient. This assessment includes not only the pattern and severity of gastrointestinal symptoms, but also the perceived eff ect of these symptoms on daily life, as well as psychological comorbidity and the presence of extra-intestinal symptoms. ${ }^{12}$ Diagnostic tests are, largely, not helpful for treatment decisions, ${ }^{13}$ with the potential exception of transit time measurement, ${ }^{14}$ anorectal physiology tests, ${ }^{15}$ hydrogen breath tests for carbohydrate malabsorption and small bowel bacterial overgrowth, ${ }^{16}$ and tests for bile acid malabsorption ${ }^{17}$ in selected groups of patients.

The fi rst step in the management approach for patients with symptoms compatible with IBS is to make a confi dent diagnosis and to involve the patient in the diagnostic strategy. For most patients with symptoms that meet the Rome IV diagnostic criteria, the number of diagnostic tests can be quite limited. ${ }^{1}$ Exceptions to this general diagnostic principle include patients who present with alarm features, such as weight loss, signs of gastrointestinal bleeding, fever, or onset of symptoms in patients older than 50 years, and patients who report diarrhoea as their predominant symptom, when more diff erential diagnoses should be considered and ruled out before an IBS diagnosis is made $^{1}$ (reviewed in more detail by Chey and colleagues ${ }^{10}$ and Enck and colleagues ${ }^{13}$ ).

Once a diagnosis of IBS is made, thorough information about the disorder should be given to patients, because explanation and reassurance constitute a basic principle of disease management. ${ }^{18}$ This explanation should include the following components: information that IBS is a very prevalent condition; a thorough explanation of the diagnosis and the underlying mechanisms of the disease; an explanation about the multicomponent character of IBS, and the bidirectional relationship between the brain and the gut; discussion about the effect of both psychological and physical stress, and cognitive and behavioural factors on symptom generation; information about the chronic but benign nature of IBS (ie, there is no increased risk for medical complications or development of other, more medically severe gastrointestinal diseases such as colon cancer); and discussion about the association between fluctuations in symptom pattern and severity with external and internal stress factors. ${ }^{18}$ For a substantial proportion of patients, this is suffi cient and no further treatment is needed. Some patients might need more extensive information and education than others, and structured patient education in group settings has proven to be useful. ${ }^{19}$ General dietary and lifestyle advice, including increased physical activity, is often offered at an early stage. ${ }^{20-22}$ For patients who do not respond to general dietary advice (panel), more specific dietary advice can be offered, such as diets restricted in poorly absorbed carbohydrates (FODMAPs [fermentable oligosaccharides, disaccharides, monosaccharides, and polyols]) or exclusion of other foods thought to trigger or worsen symptoms. For these more elaborate diets, a dietitian should be involved to

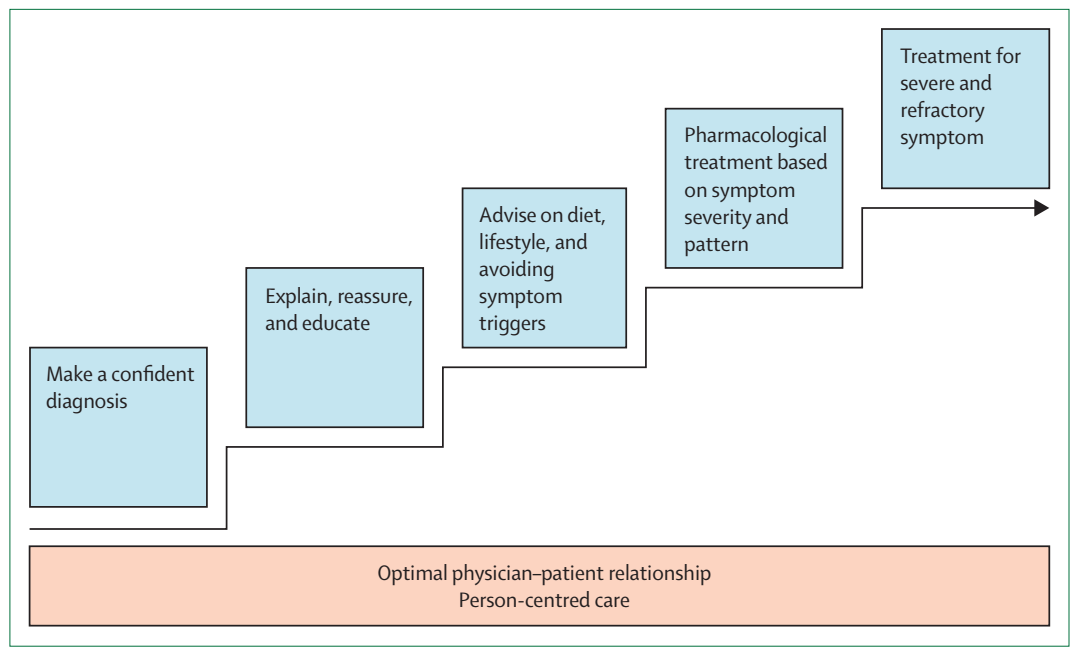

Figure 1: General treatment and symptom management principles for patients with irritable bowel syndrome

Panel: Principles of dietary management of $\mathrm{IBS}^{21}$

- Promote healthy eating and lifestyle

- Evaluate and modify: intake of alcohol, caffeine, fat, spicy food, and gas-producing foods, meal size, and number of meals per day

- Assess possibility of food intolerances

- Restrict milk and dairy products

- Modify dietary fibre

- FODMAPs-restriction, reintroduction, and long-term self-management

- Use probiotic products

FODMAPs=fermentable oligosaccharides, disaccharides, monosaccharides, and polyols

make sure that nutritional adequacy is not jeopardised. A gluten-free diet has been advocated by some, but evidence supporting this approach in IBS is incomplete and no general recommendations can be made. ${ }^{21}$ The addition of a probiotic could be considered since some of these products can improve IBS symptoms, but it is unclear which patients will benefit and whether there are differences between existing products, since no head-to-head comparisons exist. ${ }^{23}$

\section{Symptom-based management}

If conservative measures do not reduce symptoms to an acceptable level, the next step is to choose a pharmaceutical treatment on the basis of the predominant symptom and presence of other symptoms (figure 2). Different treatment options are used depending on whether patients have diarrhoea or constipation as their predominant symptom. However, some patients do not consider disturbance of their bowel habits to be their predominant symptom; rather, abdominal pain or bloating predominate and govern the treatment choice. For almost all drugs available for IBS, the overall therapeutic gain over placebo is modest, mostly ranging 


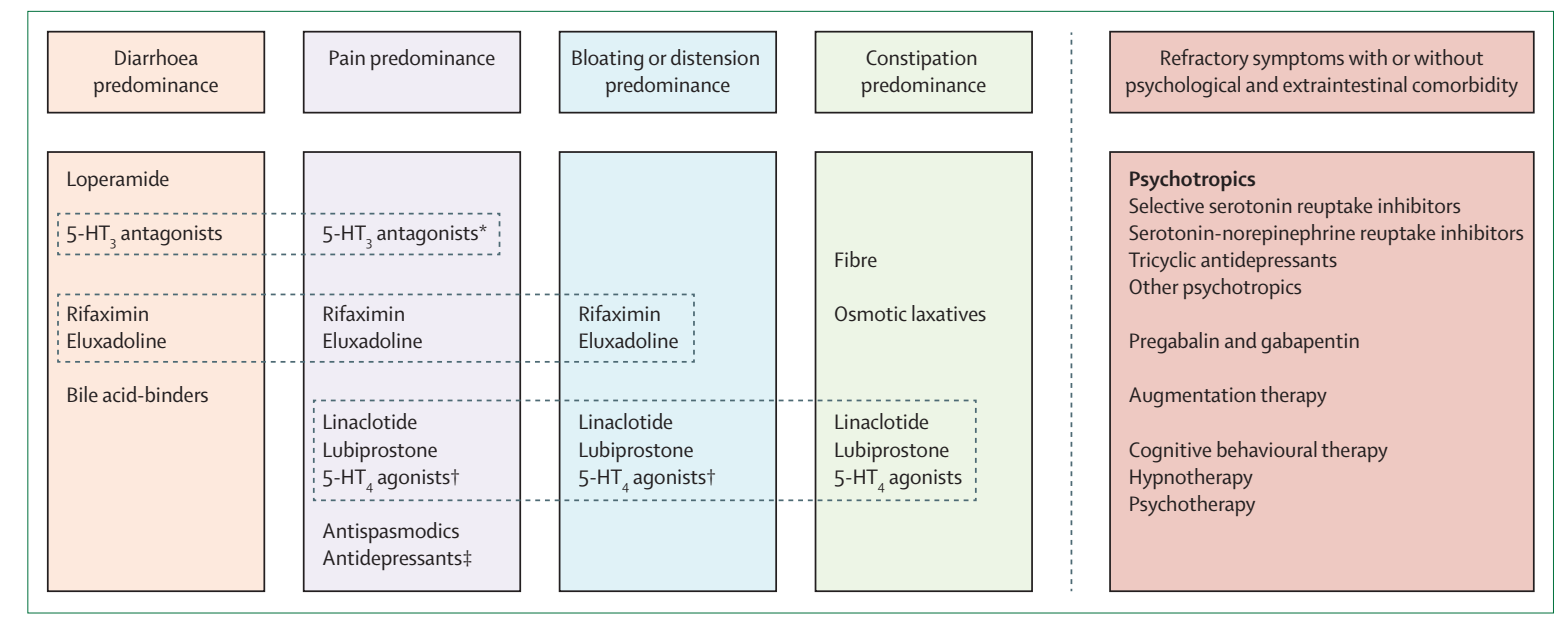

Figure 2: Symptom-based management, targeting predominant symptoms or a combination of symptoms

Overview of treatment options for patients with irritable bowel syndrome, based on the predominant symptoms. Some treatments can be used to target several symptoms, which is indicated in the figure. SSRIs=selective serotonin reuptake inhibitors. SNRIs=serotonin-norepinephrine reuptake inhibitors. ${ }^{*}$ Only alosetron and ramosetron, and not ondansetron, improved pain. †Tegaserod is not available, except for in emergency situations; prucalopride is indicated for chronic constipation, and improves pain and bloating in patients with constipation. ¥Primarily tricyclic antidepressants and serotonin-norepinephrine reuptake inhibitors have analgesic effects.

between $8 \%$ and $20 \%$, but the therapeutic gain could be larger for individual symptoms. Not only are pharmacological treatments considered at this stage, but also more advanced dietary strategies, such as a low FODMAP diet, which is useful in a substantial proportion of patients. However, no clear guidance exists regarding patient selection and how to use more advanced dietary strategies in the long term, and concerns have been raised about the potentially harmful effects of this type of diet on microbiota composition. ${ }^{21}$ Moreover, it is not clear whether a low FODMAP diet is superior to traditional IBS dietary advice based on national guidelines (eg, National Institute for Health and Care Excellence guidelines). ${ }^{21,24}$ Guidelines published in 2016 by the British Dietetic Association ${ }^{21}$ provide more details about dietary management of IBS.

\section{IBS with diarrhoea predominance}

In patients with IBS who have diarrhoea as their predominant symptom, loperamide is the most common first-line option, whereas the choice of second-line treatment differs depending on local availability and preferences.

\section{Loperamide}

Loperamide is available as an over-the-counter drug in most countries. It is a $\mu$-opioid receptor agonist acting in the myenteric plexus of the gut. Its antidiarrhoeal effects are well established: it slows transit through the small and large intestine. ${ }^{25} \mathrm{~A}$ few studies of loperamide in IBS have been done, mainly showing positive effects on stool frequency and consistency, as well as urgency, but its effect on other symptoms is negligible. ${ }^{26}$ It can be used on an as-needed basis, but a substantial proportion of patients with IBS take the drug at a fixed dose to prevent diarrhoea episodes.

\section{5- $\mathrm{HT}_{3}$ receptor agonists}

Serotonin is one of the most important neurotransmitters in the gastrointestinal tract. Antagonism of the $5-\mathrm{HT}_{3}$ receptor in the gastrointestinal tract has been shown to slow small and large bowel transit and to reduce gastrointestinal sensitivity. In line with these physiological effects, alosetron, a $5-\mathrm{HT}_{3}$ receptor antagonist, improves the key symptoms in female patients with diarrhoea-predominant IBS: pain and discomfort, diarrhoeal symptoms, and bloating. ${ }^{26,27}$ Alosetron was introduced in the second half of the $1990 \mathrm{~s}$, withdrawn from the market in 2000 because of serious side-effects, and then reintroduced in 2002 with restricted availability. The most important side-effects are constipation (10-43\%) and ischaemic colitis (about $0 \cdot 2 \%){ }^{27}$ Compared with the dosage regimen initially proposed, a 2007 study $^{28}$ showed that a dose of $0.5 \mathrm{mg}$ or $1 \mathrm{mg}$ once daily is as efficacious as $1 \mathrm{mg}$ twice daily. Ramosetron, a drug with a chemical structure similar to alosetron, is available in Asia and is efficacious in male and female patients with IBS with diarrhoea predominance. ${ }^{29,30}$ Like alosetron, it improves abdominal pain, bloating, and diarrhoeal symptoms. However, by contrast with alosetron, ramosteron has not been associated with risk of developing ischaemic colitis. ${ }^{29,30} 5-\mathrm{HT}_{3}$ antagonists are widely used to treat chemotherapy-induced nausea. One of the drugs most frequently used for this indication, ondansetron, improved some of the most intrusive symptoms in patients with IBS with predominant diarrhoea-loose stools, frequency, and urgency-but did not have a significant effect on abdominal pain. ${ }^{31}$

\section{Rifaximin}

Patients with IBS might have alterations in gut microbiota composition and function, leading investigators to assess use of antibiotics to treat symptoms. ${ }^{32}$ 
Rifaximin is an oral, minimally absorbed antibiotic that has been tested in patients with non-constipated IBS; after a 2 week treatment period, a higher proportion $(9 \%)$ of rifaximin-treated patients $(550 \mathrm{mg}$ three times daily) reported adequate relief of their overall symptoms than did placebo-treated patients..$^{33}$ Of the individual symptoms, improvements were seen in loose stools, abdominal pain, and bloating. One problem is that the eff ect gradually disappears and re-treatment is necessary in a large proportion of patients to retain symptom improvement. Re-treatment up to two times is approved by the US Food and Drug Administration, on the basis of fi ndings from a recent re-treatment trial. $^{34}$ No serious side-eff ects were reported in published trials. ${ }^{35}$

\section{Eluxadoline}

Eluxadoline is a peripherally acting, mixed agonist of $\mu$-opioid and $\kappa$-opioid receptors and a $\delta$-opioid receptor antagonist, with minimal oral bioavailability. Preclinical animal studies suggest that it reduces visceral hypersensitivity without complete inhibition of gastrointestinal motility. ${ }^{36}$ It has been approved in the USA for treatment of IBS with diarrhoea since 2015, and has just been approved in Europe. Regulatory approval was based on two large clinical trials in which eluxadoline was effective in simultaneously relieving abdominal pain and diarrhoea, and had a positive effect on bloating, in men and women with IBS with diarrhoea. The most common side-effects were nausea, constipation, and abdominal pain, and the most concerning side-effect was development of mild pancreatitis and sphincter of Oddi spasm with elevated concentrations of liver enzymes in a few patients who received the drug. ${ }^{37}$ On this basis, patients with a history of gallbladder obstruction, sphincter of Oddi spasm, pancreatic or liver disease, or alcohol overconsumption should not receive eluxadoline, and patients without a gallbladder are advised to take $75 \mathrm{mg}$ twice daily rather than the standard dose of $100 \mathrm{mg}$ twice daily.

\section{Bile acid-binding agents}

A substantial proportion of patients with IBS with diarrhoea have increased colonic bile acid exposure, ${ }^{17}$ which could explain their diarrhoeal symptoms, and can be treated with bile-acid-binding drugs. ${ }^{38}$ The presence of bile acid diarrhoea can be determined with the ${ }^{75}$ SeHCAT ( ${ }^{75}$ selenium homocholic acid taurine) test or measurement of serum ( $7 \alpha$-hydroxy-4-cholesten-3-one) $\mathrm{C} 4$, but because these tests are not widely available, clinicians often treat with bile-acid-binding drugs for 2-4 weeks to judge relevance. No large randomised controlled trials of bile-acid-binding drugs have been done in patients with IBS with diarrhoea, but small pilot and open-label studies suggest efficacy in treating diarrhoeal symptoms in these patients, and that they can be used as a second-line treatment option. ${ }^{1739}$
Most commonly, these drugs are started with a low dose (eg, cholestyramine 2-4 g daily) and gradually increased every 2-3 days until the desired effect on bowel habits (less frequent and more firm stools) is seen. This approach also seems to reduce the incidence and intensity of side-effects such as nausea and upper gastrointestinal symptoms. The newer bile-acid-binding drugs, colestipol (5 g initially, gradually increased up to a maximum of $30 \mathrm{~g}$ daily) and colesevelam (normally $3.75 \mathrm{~g}$ daily), have fewer side-effects than does cholestyramine. However, they are less well documented in the treatment of diarrhoea and more expensive, and can therefore serve as second-line options in case of poor tolerability of cholestyramine. ${ }^{38,40}$ Importantly, bile-acid-binding drugs should be taken at a different time of day than other drugs, since they can bind not only bile acids but also other drugs and thereby reduce their bioavailability.

\section{Other drugs}

Use of anti-inflammatory and antihistamine drugs and mast-cell blockage has been proposed on the basis of the potential role of local allergy-like reactions and low-grade immune activation in the gastrointestinal tract. However, the literature does not provide sufficient support for the use of these drugs in a clinical setting; further treatment trials are needed. ${ }^{41-44}$

\section{Combination of drugs}

Because most of the pharmaceutical treatments proposed for IBS with diarrhoea target different mechanisms, combining drugs to improve efficacy seems reasonable. Although this approach has not been formally tested in clinical trials, it is sometimes used successfully in clinical practice to reduce symptoms in patients with IBS with diarrhoeal symptoms that are refractory to therapy.

\section{IBS with constipation predominance}

In patients with IBS with constipation as their predominant symptom, fibre or osmotic laxatives are the most common first-line options, whereas linaclotide and lubiprostone are second-line treatment options in patients who do not respond adequately to first-line treatment. Prucalopride, although only formally approved for chronic constipation, can be considered in patients with IBS when constipation is clearly the predominant symptom.

\section{Fibre}

Patients with IBS are often advised to increase their intake of dietary fibre to improve bowel function, particularly constipation. On one hand, insoluble fibres (eg, wheat bran) provide little benefit and could worsen symptoms, especially bloating and abdominal distension. ${ }^{45}$ On the other hand, soluble fibres (eg, psyllium and ispaghula husk) were shown in recent 
meta-analyse ${ }^{26,46}$ to provide some benefit in patients with IBS, associated with relief of constipation. Therefore, because soluble fibres are inexpensive and have no serious side-effects, they can be recommended as a first-line treatment option in patients with IBS with constipation predominance. Patients should start with a low dose (eg, 3-4 g daily) that can be gradually increased, to minimise side-effects.

\section{Osmotic laxatives}

Polyethylene glycol is an established treatment for patients with chronic constipation, ${ }^{47}$ and trial data also support its use in patients with IBS with constipation. However, it only improves constipation symptoms, such as stool frequency and consistency, and severity of straining, but has no effect on abdominal pain. ${ }^{48}$ Since polyethylene glycol is inexpensive, widely available, and is not associated with any severe side-effects, it can be recommended as a first-line treatment option for patients with IBS with predominant constipation, although it can cause loose stools, bloating, and gas symptoms. Moreover, the patient should be informed that an effect can be expected only on constipation, and most likely not on other IBS-related symptoms (which is also the recommended information strategy for other treatment options effective only for specific disease symptoms)

\section{Secretagogues}

Linaclotide is a minimally absorbed guanylate cyclase C agonist. Activation of this receptor leads to increased fluid secretion into the gut lumen, and probably also to reduced gut sensitivity. Consistent with these effects on gut physiology, linaclotide improves constipation, abdominal pain, and bloating in men and women with IBS with constipation. ${ }^{49}$ Importantly, looser and more frequent stools can be seen during the first week of treatment, but it can take several weeks for the positive effect on pain and bloating to reach its maximum. Diarrhoea is the most common side-effect, reported by up to $20 \%$ of patients, but a substantially smaller proportion discontinue the treatment because of diarrhoea. ${ }^{50,51}$ The recommended dose for patients with IBS is $290 \mu \mathrm{g}$ once daily (the recommended starting dose for chronic idiopathic constipation is $145 \mu \mathrm{g}$ once daily), and it should be taken 30 to $60 \mathrm{~min}$ before the first meal of the day to reduce the risk of diarrhoea.

Lubiprostone activates type- 2 chloride channels (chloride channel protein 2) in the gastrointestinal tract, which enhances fluid secretion and thereby accelerates transit. Additionally, some studies suggest that lubiprostone improves gut barrier function. ${ }^{52}$ In randomised controlled trials, lubiprostone provided global improvement of symptoms in patients with IBS with constipation; individually, abdominal pain and discomfort, bloating, stool consistency and frequency, and severity of straining all showed greater improvement with lubiprostone than with placebo. ${ }^{53}$ Long-term follow-up studies show sustained efficacy and safety over 1 year of treatment with lubiprostone..$^{54}$ Diarrhoea and nausea are the most common side-effects. Nausea is dose-dependent and can be reduced if lubiprostone is taken with meals. The recommended dose in IBS with constipation is $8 \mu \mathrm{g}$ twice daily, as opposed to $24 \mu \mathrm{g}$ twice daily in patients with chronic constipation or opioid-induced constipation.

\section{$5-\mathrm{HT}_{4}$ receptor agonists}

5- $\mathrm{HT}_{4}$ receptors are important for peristaltic activity in the gastrointestinal tract and, therefore, $5-\mathrm{HT}_{4}$ agonists are potent prokinetic agents. Tegaserod is a $5-\mathrm{HT}_{4}$ agonist and $5-\mathrm{HT}_{2 \mathrm{~B}}$ antagonist that was approved for treatment of IBS with constipation in the USA in 2002. Studies showed that it had the most consistent effects on bowel habit. However, tegaserod was removed from the market in 2007 because of concerns about cardiovascular side-effects, and has thereafter been available only in emergency situations in a few countries. ${ }^{55}$ Prucalopride is a selective $5-\mathrm{HT}_{4}$ agonist approved for chronic constipation in patients who have not responded to treatment with laxatives, and the treatment goal is to improve stool frequency and consistency. ${ }^{56}$ It has not been formally tested in patients with IBS, but in patients with chronic constipation, positive effects were also seen on symptoms such as bloating and abdominal pain..$^{57}$ Because functional bowel disorders are not discrete disorders and exist on a continuum ${ }^{1}$ use of prucalopride seems logical when constipation is the predominant symptom (hence bordering on chronic idiopathic constipation). The recommended dose is $2 \mathrm{mg}$ once daily, or $1 \mathrm{mg}$ once daily in patients older than 65 years. The most common and problematic side-effects are diarrhoea and headaches. Side-effects can appear during the first week of treatment, but often disappear subsequently, which is important to tell to the patient.

\section{Combination of drugs}

As is the case for IBS with diarrhoea predominance, most pharmaceutical treatments proposed for IBS with constipation predominance target different mechanisms so combining these drugs to improve effectiveness seems reasonable. Although this approach has not been tested formally in clinical trials, it is sometimes used successfully in clinical practice to reduce symptoms in patients with IBS with constipation that is refractory to therapy.

\section{IBS with pain predominance}

In patients with IBS with pain as their predominant symptom, the associated bowel habit is important when deciding on a therapeutic strategy, since some of the drugs targeting pain also affect bowel habits. Antispasmodics and tricyclic antidepressants are the most widely 
used pharmacological treatments for IBS with pain predominance. However, several of the drugs discussed previously for IBS with diarrhoea or constipation predominance also reduce pain (and bloating; figure 2), ${ }^{33,37,50,51,53,57}$ so these are also valuable options in some patients, depending on the associated bowel habit.

\section{Antispasmodics}

Antispasmodics have been used for IBS for many years since smooth muscle spasms are thought to contribute to IBS pain. The mechanisms of action of antispasmodics differ, but mainly involve anticholinergic or calciumblocking properties, leading to smooth muscle relaxation, and thus reducing pain. ${ }^{58}$ Several, mainly older, trials have assessed the effects of different antispasmodics in IBS, but the quality of many of these trials is suboptimal. However, several meta-analyses have found antispasmodics as a group to be superior to placebo for IBS, particularly in treatment of pain. ${ }^{26,59,60}$ Availability of antispasmodics differs between regions, and although this class of drugs seems to be safe and serious side-effects are rare, the usefulness of many of the available drugs is limited by side-effects, mainly related to their anticholinergic properties. Antispasmodics are frequently used as a first-line treatment for patients with IBS with pain as the predominant symptom, and are often used episodically during periods where pain is particularly severe. On the basis of the side-effect profile of antispasmodics with anticholinergic effects, these drugs seem to be preferable in patients with pain and diarrhoea. Peppermint oil is also thought to have smooth-muscle-relaxing properties, but might attenuate visceral hypersensitivity. ${ }^{26}$ In meta-analyses, ${ }^{26,59,60}$ the effects of peppermint oil seem to be favourable, but the quality of included studies is poor. However, a new delivery system of peppermint oil has been approved in the USA on the basis of promising results in a small clinical trial, ${ }^{61}$ which showed a positive effect on overall IBS symptoms, as well as individual symptoms such as pain, bloating, abdominal distension, and urgency.

\section{Antidepressants}

Since anxiety and depression are frequent comorbidities in patients with IBS, antidepressants have been introduced as treatment options. ${ }^{62}$ However, antidepressants are just as frequently used to target pain as they are for treating comorbid anxiety and depression. ${ }^{63}$ Several clinical trials have shown the usefulness of antidepressants to improve global IBS symptoms, and this has been substantiated in a meta-analysis, ${ }^{64}$ with a number-needed-to-treat of four for both tricyclic antidepressants and selective serotonin reuptake inhibitors (SSRIs). Based on their known effects on pain reduction, tricyclic antidepressants are the logical choice for patients with pain predominance; serotoninnorepinephrine reuptake inhibitors (SNRIs) are usually a second-line option, although they have not formally been studied in IBS. ${ }^{63}$ Results of several studies show the efficacy of tricyclic antidepressants to improve pain in IBS, and lower doses can be used for pain than for depression..$^{64,65}$ Side-effects, mainly related to anticholinergic and antihistamine effects, limit their use in some patients. Consistent with the recommendations for antispasmodics, they could be more useful and better tolerated in patients with pain accompanied by diarrhoea, than in patients with constipation.

SNRIs are beneficial for treatment of pain in other disorders such as fibromyalgia and peripheral diabetic neuropathic pain, with more compelling evidence for duloxetine (usual dose $60 \mathrm{mg}$ daily) ${ }^{66}$ than for venlafaxine (usually 75-225 mg daily). ${ }^{67}$ In clinical practice, SNRIs are also used to treat pain in patients with functional gastrointestinal disorders, although formal scientific evidence from randomised controlled trials in IBS is absent. $^{63}$ Since SNRIs (like SSRIs) do not have anticholinergic side-effects, they can be used in patients with constipation. Although SSRIs improve overall symptoms in patients with IBS, they do not have profound analgesic effects, and are therefore less promising options for patients with pain predominance. ${ }^{62,63}$

When treating patients with IBS with antidepressants, it is of great importance to stress that their peripheral and neuromodulatory effects are just as important as (and sometimes more important than) their antidepressant effects. Side-effects tend to appear early in treatment and often dissipate thereafter, whereas the positive effect on gastrointestinal symptoms might occur later. Therefore, it is important to inform patients about this, and to provide close support when initiating these treatment options. To increase the likelihood of a successful outcome, treatment should be started with a low dose that is gradually increased over the first month (or months) until the target dose is reached..$^{62,63}$

\section{Combination of drugs}

Consistent with previous recommendations in this Review, it seems reasonable to combine antidepressants or antispasmodics with drugs that target the predominant bowel habit to improve efficacy, on the basis of different and complementary modes of action. This approach has not been tested formally in clinical trials, but is often used successfully in clinical practice. Furthermore, a recent 2016 study suggests that pain, and potentially other key IBS symptoms of IBS, might be caused by histamine $\mathrm{H} 1$ receptor-mediated visceral hypersensitivity, and favourably treated with a histamine H1 receptor antagonist (ebastine), but further trials are needed before this treatment approach can be recommended for clinical use. ${ }^{44}$

\section{IBS with bloating and distension predominance}

There are no effective drugs that specifically target bloating and abdominal distension in IBS. Dietary strategies include avoiding intake of gas-producing 
foods (eg, cabbage and onion) reducing intake of (especially insoluble) dietary fibre, and restricting intake of foods containing FODMAPs, particularly after demonstration of carbohydrate (eg, lactose or fructose) malabsorption. ${ }^{21}$ Probiotics are often used to reduce bloating and abdominal distension, since some probiotic products seem to improve these symptoms. ${ }^{23,68}$ Moreover, several newer drugs, such as rifaximin, ${ }^{33}$ eluxadoline,${ }^{37}$ linaclotide,${ }^{50,51}$ lubiprostone,${ }^{53}$ and, to some extent, $5-\mathrm{HT}_{4}$ agonists, ${ }^{57}$ improved bloating and abdominal distension in pivotal large-scale clinical trials, so use of these drugs to target bloating and the relevant bowel habit abnormality is a valid approach. Evidence also suggests that visible abdominal distension can be caused by descent of the diaphragm and protrusion of the anterior abdominal wall, which can be corrected with electromyography-guided, respiratorytargeted biofeedback treatment. ${ }^{69}$ However, this treatment option is still only available in a few expert centres worldwide.

\section{Treatment-refractory, psychological, and extra-intestinal symptoms}

Unfortunately a substantial proportion of patients have IBS that is refractory to the treatment proposals outlined, and a large proportion of these patients also have comorbid psychological symptoms or a wide range of extra-intestinal symptoms. ${ }^{7.8}$ In these patients, targeting the central nervous system with psychotropic drugs or psychological treatments is a valid approach, and often improves symptoms. ${ }^{62,63,70}$ However, it is important to inform patients that these treatments can also have profound positive effects on gastrointestinal symptoms.

\section{Psychotropics}

In patients with refractory symptoms, the goal of treatment with antidepressants is not only to reduce pain, but also to improve other IBS symptoms, comorbid anxiety, depression, and extra-intestinal symptoms; the combination of symptoms often determines the choice of pharmaceutical agent. ${ }^{62,63}$ Tricyclic antidepressants (most often used at low doses) are most helpful when pain (both gastrointestinal and extra-intestinal) is a prominent feature, particularly in patients with diarrhoea rather than constipation. ${ }^{64,65}$ The sedating effect of these drugs could benefit patients who have sleeping difficulties (eg, tricyclic antidepressants taken at bedtime). SSRIs seem to be useful in patients with comorbid anxiety, depression, or phobic features, and although they are not particularly useful for patients in whom pain is predominant, improvement of overall symptoms also supports the use of SSRIs in patients without psychiatric comorbidity. ${ }^{62-64}$ Considering the central role of serotonin and norepinephrine in descending pain modulatory pathways, and their mild side-effect profile, SNRIs are logical class of drugs to treat comorbid extra-intestinal pain in patients with IBS, with or without comorbid depression. ${ }^{66,67}$ Overlap with functional dyspepsia and other functional upper gastrointestinal syndromes, including nausea, is common in IBS. Therefore, use of psychotropic drugs with positive effects on gastric accommodation (buspirone), and anorexia, nausea, and weight-loss (mirtazapine), as well as antidepressant (mirtazapine) and anxiolytic effects (buspirone), could be useful in patients with overlapping upper and lower gastrointestinal symptoms and comorbid psychiatric disorders that are refractory to therapy. ${ }^{63}$

\section{Pregabalin and gabapentin}

Pregabalin and gabapentin are thought to reduce pain by binding with high affinity to $\alpha 2 \delta$ subunits of voltage-gated calcium channels. ${ }^{71}$ These drugs are widely used for different painful conditions, such as post-herpetic neuralgia, diabetic neuropathy, peripheral neuropathy, and fibromyalgia; they also have anxiolytic effects and are recommended as adjunctive therapy for generalised anxiety disorder. ${ }^{72,73}$ Limited evidence supports the usefulness of pregabalin and gabapentin in IBS, but they are sometimes used in patients who are refractory to therapy, for whom pain or anxiety are important and dominant symptoms. Pregabalin and gabapentin are also sometimes used in combination with other psychotropic drugs.$^{63}$ Evidence suggests that pregabalin and gabapentin reduce visceral hypersensitivity in IBS, but no clinical trials have evaluated their effects on symptoms. ${ }^{74,75}$ The dose recommended for pregabalin, when treating pain, is between 150 and $600 \mathrm{mg}$ per day.

\section{Augmentation therapy}

The concept of augmentation therapy refers to treatment combinations involving multiple psychotropic drugs, or a combination of psychological treatment with one or more of these drugs. This approach can be used in patients not responding sufficiently to one specific class of drugs, and when increasing the dose does not seem to the best option because of the risk of side-effects. When individual pharmacological treatments are combined, the doses can be lower than when they are used individually. The goal of this approach is to achieve synergistic treatment effects and improve tolerance by targeting different receptors. The treating physician using this approach should be familiar with which receptors are targeted by different drugs, and the risk of side-effects with the individual drugs and drug combinations, including the risk of development of serotonin syndrome (hyperreflexia, spontaneous clonus, muscle rigidity, and fever) due to negative synergistic effects. ${ }^{62,63}$

\section{Cognitive behavioural therapy}

Cognitive behavioural therapy (CBT) is the most widely studied form of psychological intervention in IBS, and results of meta-analyses showed a number-needed-to-treat 
of three for overall improvement of IBS symptoms, ${ }^{64}$ with good short-term and long-term efficacy. ${ }^{70}$ The basis for CBT is the concept that maladaptive thoughts are the causes of psychological disorders such as anxiety and depression, which in turn cause or exacerbate physical symptoms. CBT aims to help the patient to recognise maladaptive thoughts and self-defeating behaviour patterns that adversely affect functioning, experience of symptoms, and mental wellbeing. ${ }^{76}$ CBT can be administered on an individual basis (often six to 12 sessions), in a group setting, self-administered, in a minimal-contact fashion, or via the internet-all of which have demonstrated effi cacy in patients with IBS. ${ }^{64,76}$ In clinical practice, the limited availability of therapists skilled in applying CBT to gastrointestinal problems means that it is often reserved for patients with refractory symptoms. However, CBT seems to also be effi cacious in patients without excessive comorbidity. Therefore, the development of less resource-demanding forms of CBT (self-administered, minimal contact, or internet-delivered CBT) could provide this treatment to larger groups of patients. ${ }^{7,78}$

\section{Hypnotherapy}

Hyponotherapy was pioneered in the mid-1980s by a group in Manchester, UK, who successfully treated therapy-refractory IBS with gut-directed hypnotherapy. ${ }^{79}$ Several subsequent trials confirmed the efficacy of this treatment option, and meta-analyses demonstrated good short-term and long-term efficacy, with a number-needed-to-treat of four to improve overall IBS symptoms..$^{6470}$ Results in children with functional gastrointestinal disorders seem to be even better than in adults with IBS. ${ }^{80}$ Most trials of hypnotherapy for IBS have used six to 12 sessions over 3 months. In gut-directed hypnotherapy, targeted verbal suggestions and therapeutic imagery to encourage improvement in symptoms are used to regulate smooth muscle activity, reduce the effect of stress on gastrointestinal symptoms, reduce gut pain perception and attention to symptoms, and increase the patient's sense of control over symptoms. ${ }^{7}$ As with CBT, the limited availability of therapists trained in this special application of hypnosis is a problem, although group hypnotherapy has been shown to be as efficacious as individual hypnotherapy, which could increase therapist availability. ${ }^{81}$

Psychodynamic and interpersonal psychotherapy

Fewer studies have been published using psychodynamic and interpersonal psychotherapy in IBS than with CBT and hypnotherapy, but these forms of psychotherapy can also benefit patients with IBS. ${ }^{64}$ The aim of psychodynamic psychotherapy is to reduce severity of symptoms by helping the patient understand and gain insight into unconscious processes that might cause those symptoms. By contrast, the aim of interpersonal psychotherapy is to help the patient to learn more adaptive ways of managing

\section{Search strategy and selection criteria}

We searched PubMed using the terms "irritable bowel syndrome" and "treatment", and we focused principally on recent meta-analyses, reviews, and society recommendations published between 2006 and 2016, regarding treatment and management of IBS, to identify individual treatment and management trials of greatest relevance for this Review.

interpersonal conflict and stress through an examination of the relationship between the patients and significant individuals in their life. ${ }^{76}$

\section{Other psychological treatments}

Other psychological treatments have been tested in patients with IBS, but have less evidence supporting their efficacy. ${ }^{76}$ For example, relaxation therapy has been tested in several small trials, but was not found to be superior to the control condition in improving overall IBS symptoms in a 2014 meta-analysis. ${ }^{64}$ Mindfulness is another promising alternative, and in a 2011 trial, improved bowel symptoms and quality of life, and reduced distress in female patients with IBS compared with a support group; this effect persisted for up to 3 months. ${ }^{82}$ Unfortunately, there are no direct comparisons between different psychological treatments, so availability and knowledge about local service providers often determines choice of treatment. However, CBT seems to be the best choice for patients with maladaptive thoughts about their symptoms, or when treating psychological comorbidity is an important treatment goal. If extra-intestinal physical symptoms are prominent, hypnotherapy seems to be one of the best options on the basis of its ability to improve gastrointestinal as well as extra-intestinal symptoms. ${ }^{76}$

\section{Conclusion}

A stepwise management approach is advocated for patients with IBS. For most patients, general management principles are sufficient: making a confident diagnosis (including providing information about the name of the diagnosis, ideally in written form); explaining the most likely mechanism of the patient's symptoms and reassuring them that a life-threatening illness is unlikely to be the cause of symptoms; and identifying and modifying important dietary and lifestyle triggers. However, many patients continue to have symptoms and are not satisfied solely with this approach. For these patients, pharmacotherapy based on the predominant symptoms and combination of symptoms is the next step. $^{83}$ For patients who prove refractory to pharmacological treatment of predominant symptoms, a combination of drugs should be considered, and the use of psychotropic drugs and psychological treatment approaches is often effective, either on their own or in combination. Last but not least, it should be emphasised 
that the key to success when treating patients with IBS is a good physician-patient relationship, and use of person-centred care principles.

\section{Contributors}

MS is the guarantor of article. MS performed the literature search, and wrote the initial manuscript. All authors agreed on the outline of the Review, and revised the manuscript. All authors have approved the final version of the Review.

\section{Declaration of interests}

MS has received unrestricted research grants from Danone and Ferring Pharmaceuticals, and served as a consultant/advisory board member for AstraZeneca, Danone, Nestlé, Chr Hansen, Almirall, Allergan, Albireo, Glycom, and Shire, and as a speaker for Tillotts, Takeda, Shire, and Almirall. HT has served as consultant/advisory board member for Almirall, Danone, and Shire. OSP has received salary support from research grants from the US National Institutes of Health (NIH), Takeda Pharmaceuticals, and Salix Pharmaceuticals, a consulting agreement with Ironwood Pharmaceuticals, and an educational grant provided by Takeda Pharmaceuticals, and has received a speaker honorarium in an educational program supported by Ironwood Pharmaceuticals and Takeda Pharmaceuticals. WEW has received research grants from the US NIH, Takeda, Ironwood, Salix, and the Rome Foundation, has served as a consultant to Biomerica USA, Ono Pharmaceuticals, and Ferring, and has received unrestricted educational grants from Takeda and Ferring.

\section{Acknowledgments}

The authors' research activities are supported by US National Institute of Diabetes and Digestive and Kidney Diseases (grant RO1 DK31369) (WEW), the Swedish Medical Research Council (grants 13409, 21691, and 21692) (MS), AFA Insurance (MS), an unrestricted grant from Ferring Pharmaceuticals (MS), and by the Faculty of Medicine, University of Gothenburg, Sweden (HT and MS).

\section{References}

1 Lacy BE, Mearin F, Chang L, et al. Bowel disorders. Gastroenterology 2016; 150: 1393-407.

2 Lovell RM, Ford AC. Global prevalence of and risk factors for irritable bowel syndrome: a meta-analysis. Clin Gastroenterol Hepatol 2012; 10: 712-21.

3 Canavan C, West J, Card T. Review article: the economic impact of the irritable bowel syndrome. Aliment Pharmacol Ther 2014 40: $1023-34$

4 Ringstrom G, Abrahamsson H, Strid H, Simren M. Why do subjects with irritable bowel syndrome seek health care for their symptoms? Scand J Gastroenterol 2007; 42: 1194-203.

5 Hillila MT, Siivola MT, Farkkila MA. Comorbidity and use of health-care services among irritable bowel syndrome sufferers. Scand J Gastroenterol 2007; 42: 799-806.

6 Locke GR 3rd, Zinsmeister AR, Fett SL, Melton LJ 3rd, Talley NJ. Overlap of gastrointestinal symptom complexes in a US community. Neurogastroenterol Motil 2005; 17: 29-34.

7 Whitehead WE, Palsson O, Jones KR. Systematic review of the comorbidity of irritable bowel syndrome with other disorders: what are the causes and implications? Gastroenterology 2002; 122: $1140-56$.

8 Vu J, Kushnir V, Cassell B, Gyawali CP, Sayuk GS. The impact of psychiatric and extraintestinal comorbidity on quality of life and bowel symptom burden in functional GI disorders. Neurogastroenterol Motil 2014; 26: 1323-32.

9 Simren M, Svedlund J, Posserud I, Bjornsson ES, Abrahamsson H. Health-related quality of life in patients attending a gastroenterology outpatient clinic: functional disorders versus organic diseases. Clin Gastroenterol Hepatol 2006; 4: 187-95.

10 Chey WD, Kurlander J, Eswaran S. Irritable bowel syndrome: a clinical review. JAMA 2015; 313: 949-58.

11 Owens DM, Nelson DK, Talley NJ. The irritable bowel syndrome: long-term prognosis and the physician-patient interaction. Ann Intern Med 1995; 122: 107-12.

12 Drossman DA. Functional gastrointestinal disorders: history, pathophysiology, clinical features and Rome IV. Gastroenterology 2016; 150: 1262-79.
13 Enck P, Aziz Q, Barbara G, et al. Irritable bowel syndrome. Nat Rev Dis Primers 2016; 2: 16014.

14 Tornblom H, Van Oudenhove L, Sadik R, Abrahamsson H, Tack J, Simren M. Colonic transit time and IBS symptoms: what's the link? Am J Gastroenterol 2012; 107: 754-60.

15 Rao SS, Bharucha AE, Chiarioni G, et al. Functional anorectal disorders. Gastroenterology 2016; 150: 1430-42.

16 Simren M, Stotzer PO. Use and abuse of hydrogen breath tests. Gut 2006; 55: 297-303.

17 Bajor A, Tornblom H, Rudling M, Ung KA, Simren M. Increased colonic bile acid exposure: a relevant factor for symptoms and treatment in IBS. Gut 2015; 64: 84-92.

18 Schmulson MJ, Ortiz-Garrido OM, Hinojosa C, Arcila D. A single session of reassurance can acutely improve the self-perception of impairment in patients with IBS. J Psychosom Res 2006; 61: 461-67.

19 Ringstrom G, Storsrud S, Posserud I, Lundqvist S, Westman B, Simren M. Structured patient education is superior to written information in the management of patients with irritable bowel syndrome: a randomized controlled study. Eur J Gastroenterol Hepato 2010; 22: 420-28.

20 Johannesson E, Simren M, Strid H, Bajor A, Sadik R. Physical activity improves symptoms in irritable bowel syndrome: a randomized controlled trial. Am J Gastroenterol 2011; 106: 915-22.

21 McKenzie YA, Bowyer RK, Leach H, et al. British Dietetic Association systematic review and evidence-based practice guidelines for the dietary management of irritable bowel syndrome in adults (2016 update). J Hum Nutr Diet 2016; 29: 549-75.

22 Johannesson E, Ringstrom G, Abrahamsson H, Sadik R. Intervention to increase physical activity in irritable bowel syndrome shows long-term positive effects. World J Gastroenterol 2015; 21: 600-08.

23 Ford AC, Quigley EM, Lacy BE, et al. Efficacy of prebiotics, probiotics, and synbiotics in irritable bowel syndrome and chronic idiopathic constipation: systematic review and meta-analysis. Am J Gastroenterol 2014; 109: 1547-61.

24 Bohn L, Storsrud S, Liljebo T, et al. Diet low in FODMAPs reduces symptoms of irritable bowel syndrome as well as traditional dietary advice: a randomized controlled trial. Gastroenterology 2015; 149: 1399-407.

25 Cann PA, Read NW, Holdsworth CD, Barends D. Role of loperamide and placebo in management of irritable bowel syndrome (IBS). Dig Dis Sci 1984; 29: 239-47.

26 Ford AC, Moayyedi P, Lacy BE, et al. American College of Gastroenterology monograph on the management of irritable bowel syndrome and chronic idiopathic constipation. Am J Gastroenterol 2014; 109 (suppl 1): S2-26.

27 Andresen V, Montori VM, Keller J, West CP, Layer P Camilleri M. Effects of 5-hydroxytryptamine (serotonin) type 3 antagonists on symptom relief and constipation in nonconstipated irritable bowel syndrome: a systematic review and meta-analysis of randomized controlled trials. Clin Gastroenterol Hepatol 2008; 6: 545-55.

28 Krause R, Ameen V, Gordon SH, et al. A randomized, double-blind, placebo-controlled study to assess efficacy and safety of $0.5 \mathrm{mg}$ and $1 \mathrm{mg}$ alosetron in women with severe diarrhea-predominant IBS. Am J Gastroenterol 2007; 102: 1709-19.

29 Fukudo S, Ida M, Akiho H, Nakashima Y, Matsueda K. Effect of ramosetron on stool consistency in male patients with irritable bowel syndrome with diarrhea. Clin Gastroenterol Hepatol 2014; 12: 953-59.

30 Fukudo S, Kinoshita Y, Okumura T, et al. Ramosetron reduces symptoms of irritable bowel syndrome with diarrhea and improves quality of life in women. Gastroenterology 2016; 150: 358-66

31 Garsed K, Chernova J, Hastings M, et al. A randomised trial of ondansetron for the treatment of irritable bowel syndrome with diarrhoea. Gut 2014; 63: 1617-25.

32 Pimentel M, Park S, Mirocha J, Kane SV, Kong Y. The effect of nonabsorbed oral antibiotic (rifaximin) on the symptoms of the irritable bowel syndrome: a randomized trial. Ann Intern Med 2006 145: 557-63.

33 Pimentel M, Lembo A, Chey WD, et al. Rifaximin therapy for patients with irritable bowel syndrome without constipation. N Engl J Med 2011; 364: 22-32. 
34 Lembo A, Pimentel M, Rao SS, et al. Repeat treatment with rifaximin is safe and effective in patients with diarrhea-predominant irritable bowel syndrome. Gastroenterology 2016; published online Aug 13. DOI:10.1053/j.gastro.2016.08.003.

35 Menees SB, Maneerattannaporn M, Kim HM, Chey WD. The efficacy and safety of rifaximin for the irritable bowel syndrome: a systematic review and meta-analysis. Am J Gastroenterol 2012; 107: $28-35$.

36 Lacy BE. Emerging treatments in neurogastroenterology: eluxadoline-a new therapeutic option for diarrhea-predominant IBS. Neurogastroenterol Motil 2016; 28: 26-35.

37 Lembo AJ, Lacy BE, Zuckerman MJ, et al. Eluxadoline for irritable bowel syndrome with diarrhea. N Engl J Med 2016; 374: 242-53.

38 Mottacki N, Simren M, Bajor A. Review article: bile acid diarrhoea-pathogenesis, diagnosis and management. Aliment Pharmacol Ther 2016; published online Feb 24. DOI:10.1111/ apt.13570.

39 Camilleri M, Acosta A, Busciglio I, et al. Effect of colesevelam on faecal bile acids and bowel functions in diarrhoea-predominant irritable bowel syndrome. Aliment Pharmacol Ther 2015; 41: 438-48.

40 Wilcox C, Turner J, Green J. Systematic review: the management of chronic diarrhoea due to bile acid malabsorption. Aliment Pharmacol Ther 2014; 39: 923-39.

41 Barbara G, Cremon C, Annese V, et al. Randomised controlled trial of mesalazine in IBS. Gut 2016; 65: 82-90.

42 Lam C, Tan W, Leighton M, et al. A mechanistic multicentre, parallel group, randomised placebo-controlled trial of mesalazine for the treatment of IBS with diarrhoea (IBS-D). Gut 2016; 65: 91-9.

43 Ohman L, Simren M. Pathogenesis of IBS: role of inflammation, immunity and neuroimmune interactions. Nat Rev Gastroenterol Hepatol 2010; 7: 163-73.

44 Wouters MM, Balemans D, Van Wanrooy S, et al. Histamine receptor H1-mediated sensitization of TRPV1 mediates visceral hypersensitivity and symptoms in patients with irritable bowel syndrome. Gastroenterology 2016; 150: 875-87.

45 Bijkerk CJ, Muris JW, Knottnerus JA, Hoes AW, de Wit NJ. Systematic review: the role of different types of fibre in the treatment of irritable bowel syndrome. Aliment Pharmacol Ther 2004; 19: 245-51.

46 Moayyedi P, Quigley EM, Lacy BE, et al. The effect of fiber supplementation on irritable bowel syndrome: a systematic review and meta-analysis. Am J Gastroenterol 2014; 109: 1367-74.

47 Belsey JD, Geraint M, Dixon TA. Systematic review and meta analysis: polyethylene glycol in adults with non-organic constipation. Int J Clin Pract 2010; 64: 944-55.

48 Chapman RW, Stanghellini V, Geraint M, Halphen M. Randomized clinical trial: macrogol/PEG 3350 plus electrolytes for treatment of patients with constipation associated with irritable bowel syndrome. Am J Gastroenterol 2013; 108: 1508-15.

49 Layer P, Stanghellini V. Review article: linaclotide for the management of irritable bowel syndrome with constipation. Aliment Pharmacol Ther 2014; 39: 371-84.

50 Chey WD, Lembo AJ, Lavins BJ, et al. Linaclotide for irritable bowe syndrome with constipation: a 26-week, randomized, double-blind, placebo-controlled trial to evaluate efficacy and safety. Am J Gastroenterol 2012; 107: 1702-12.

51 Rao S, Lembo AJ, Shiff SJ, et al. A 12-week, randomized, controlled trial with a 4-week randomized withdrawal period to evaluate the efficacy and safety of linaclotide in irritable bowel syndrome with constipation. Am J Gastroenterol 2012; 107: 1714-24.

52 Raschi E, De Ponti F. Lubiprostone: pharmacokinetic, pharmacodynamic, safety and regulatory aspects in the treatment of constipation-predominant irritable bowel syndrome. Expert Opin Drug Metab Toxicol 2014; 10: 293-305.

53 Drossman DA, Chey WD, Johanson JF, et al. Clinical trial: lubiprostone in patients with constipation-associated irritable bowe syndrome-results of two randomized, placebo-controlled studies. Aliment Pharmacol Ther 2009; 29: 329-41.

54 Chey WD, Drossman DA, Johanson JF, Scott C, Panas RM, Ueno R. Safety and patient outcomes with lubiprostone for up to 52 weeks in patients with irritable bowel syndrome with constipation. Aliment Pharmacol Ther 2012; 35: 587-99.
55 Evans BW, Clark WK, Moore DJ, Whorwell PJ. Tegaserod for the treatment of irritable bowel syndrome and chronic constipation. Cochrane Database Syst Rev 2007; 4: CD003960.

56 Tack J, Quigley E, Camilleri M, Vandeplassche L, Kerstens R. Efficacy and safety of oral prucalopride in women with chronic constipation in whom laxatives have failed: an integrated analysis. United European Gastroenterol J 2013; 1: 48-59.

57 Tack J, Stanghellini V, Dubois D, Joseph A, Vandeplassche L, Kerstens R. Effect of prucalopride on symptoms of chronic constipation. Neurogastroenterol Motil 2014; 26: 21-27.

58 Annahazi A, Roka R, Rosztoczy A, Wittmann T. Role of antispasmodics in the treatment of irritable bowel syndrome. World J Gastroenterol 2014; 20: 6031-43.

59 Ford AC, Talley NJ, Spiegel BM, et al. Effect of fibre, antispasmodics, and peppermint oil in the treatment of irritable bowel syndrome: systematic review and meta-analysis. BMJ 2008; 337: a2313.

60 Ruepert L, Quartero AO, de Wit NJ, van der Heijden GJ, Rubin G, Muris JW. Bulking agents, antispasmodics and antidepressants for the treatment of irritable bowel syndrome. Cochrane Database Syst Rev 2011; 8: CD003460.

61 Cash BD, Epstein MS, Shah SM. A novel delivery system of peppermint oil is an effective therapy for irritable bowel syndrome symptoms. Dig Dis Sci 2016; 61: 560-71.

62 Grover M, Drossman DA. Psychotropic agents in functional gastrointestinal disorders. Curr Opin Pharmacol 2008; 8: 715-23.

63 Tornblom H, Drossman DA. Centrally targeted pharmacotherapy for chronic abdominal pain. Neurogastroenterol Motil 2015; 27: 455-67.

64 Ford AC, Quigley EM, Lacy BE, et al. Effect of antidepressants and psychological therapies, including hypnotherapy, in irritable bowel syndrome: systematic review and meta-analysis. Am J Gastroenterol 2014; 109: 1350-65.

65 Vahedi H, Merat S, Momtahen S, et al. Clinical trial: the effect of amitriptyline in patients with diarrhoea-predominant irritable bowel syndrome. Aliment Pharmacol Ther 2008; 27: 678-84.

66 Lunn MP, Hughes RA, Wiffen PJ. Duloxetine for treating painful neuropathy, chronic pain or fibromyalgia. Cochrane Database Syst Rev 2014; 1: CD007115.

67 Gallagher HC, Gallagher RM, Butler M, Buggy DJ, Henman MC. Venlafaxine for neuropathic pain in adults. Cochrane Database Syst Rev 2015; 8: CD011091.

68 Agrawal A, Houghton LA, Morris J, et al. Clinical trial: the effects of a fermented milk product containing Bifidobacterium lactis DN-173 010 on abdominal distension and gastrointestinal transit in irritable bowel syndrome with constipation. Aliment Pharmacol Ther 2009; 29: 104-14.

69 Barba E, Burri E, Accarino A, et al. Abdominothoracic mechanisms of functional abdominal distension and correction by biofeedback. Gastroenterology 2015; 148: 732-9.

70 Laird KT, Tanner-Smith EE, Russell AC, Hollon SD, Walker LS. Short-term and long-term efficacy of psychological therapies for irritable bowel syndrome: a systematic review and meta-analysis. Clin Gastroenterol Hepatol 2016; 14: 937-47.

71 Taylor CP. Mechanisms of analgesia by gabapentin and pregabalin-calcium channel alpha2-delta [Cavalpha2-delta] ligands. Pain 2009; 142: 13-16.

72 Tzellos TG, Papazisis G, Toulis KA, Sardeli C, Kouvelas D. A2delta ligands gabapentin and pregabalin: future implications in daily clinical practice. Hippokratia 2010; 14: 71-75.

73 Tzellos TG, Toulis KA, Goulis DG, et al. Gabapentin and pregabalin in the treatment of fibromyalgia: a systematic review and a meta-analysis. J Clin Pharm Ther 2010; 35: 639-56.

74 Gale JD, Houghton LA. Alpha 2 delta (alpha(2)delta) ligands, gabapentin and pregabalin: what is the evidence for potential use of these ligands in irritable bowel syndrome. Front Pharmacol 2011; 2: 28.

75 Houghton LA, Fell C, Whorwell PJ, Jones I, Sudworth DP, Gale JD. Effect of a second-generation alpha2delta ligand (pregabalin) on visceral sensation in hypersensitive patients with irritable bowe syndrome. Gut 2007; 56: 1218-25.

76 Palsson OS, Whitehead WE. Psychological treatments in functional gastrointestinal disorders: a primer for the gastroenterologist. Clin Gastroenterol Hepatol 2013; 11: 208-16. 
77 Lackner JM, Jaccard J, Krasner SS, Katz LA, Gudleski GD, Holroyd K. Self-administered cognitive behavior therapy for moderate to severe irritable bowel syndrome: clinical efficacy, tolerability, feasibility. Clin Gastroenterol Hepatol 2008; 6: 899-906.

78 Ljotsson B, Hedman E, Andersson E, et al. Internet-delivered exposure-based treatment vs. stress management for irritable bowe syndrome: a randomized trial. Am J Gastroenterol 2011; 106: 1481-91.

79 Whorwell PJ, Prior A, Faragher EB. Controlled trial of hypnotherapy in the treatment of severe refractory irritable-bowel syndrome. Lancet 1984; 2: 1232-34.

80 Rutten JM, Reitsma JB, Vlieger AM, Benninga MA. Gut-directed hypnotherapy for functional abdominal pain or irritable bowel syndrome in children: a systematic review. Arch Dis Child 2013; 98: $252-57$.
81 Moser G, Tragner S, Gajowniczek EE, et al. Long-term success of GUT-directed group hypnosis for patients with refractory irritable bowel syndrome: a randomized controlled trial. Am J Gastroenterol 2013; 108: 602-09.

82 Gaylord SA, Palsson OS, Garland EL, et al. Mindfulness training reduces the severity of irritable bowel syndrome in women: results of a randomized controlled trial. Am J Gastroenterol 2011; 106: $1678-88$.

83 Chang L, Lembo A, Sultan S. American Gastroenterological Association Institute technical review on the pharmacological management of irritable bowel syndrome. Gastroenterology 2014; 147: 1149-72. 